\title{
Near-field Source Localization Based on Sparse Reconstruction of Sensor-Angle Distributions
}

\author{
Yimin D. Zhang, Si Qin, and Moeness G. Amin \\ Center for Advanced Communications, Villanova University, Villanova, PA 19085, USA
}

\begin{abstract}
In this paper, we consider a problem of near-field source localization using the sensor-angle distribution (SAD) that views the source range and direction-of-arrival (DOA) information as sensor-dependent phase progression. The SAD draws parallel to quadratic time-frequency distributions and, as such, is able to reveal the changes in the spatial frequency over sensor positions. In particular, for a moderate source range, the SAD signature is of polynomial shape, thus simplifying the parameter estimation. We consider sparse arrays where the array sensors are located on a grid but with missing positions. Sparse reconstruction techniques are used to estimate the SAD in the joint space and spatial frequency domain, and the results are then mapped back to source range and DOA estimation for source localization. The effectiveness of the proposed technique is verified using simulation results.
\end{abstract}

\section{INTRODUCTION}

Localization of near-field emitting sources is an important application in many radar applications. Such problems are much more challenging than the far-field counterparts as the received signals are a coupled function of both the range and the direction-of-arrival (DOA) of each source. A number of methods have been developed to decouple these two variables for simplified computations, but they usually entail some constraints and compromised performance. For example, the fourth-order statistics based approaches require a uniform linear array (ULA) with an inter-element spacing of quarter wavelength [1], [2].

In this paper, we consider the sensor-angle distribution (SAD) that maps the source range and DOA information as sensor-dependent phase progression, and the yielding distribution presented in the joint space and spatial frequency domain resembles to the time-frequency signature for instantaneous narrowband nonstationary signals [3]. As such, the signal processing techniques established in the time-frequency domain can be readily applied for the processing of the SAD for near-field source localization. In particular, for a moderate source range, the SAD signature is of polynomial shape, thus simplifying the parameter estimation. Note that the SAD was originally developed in the form of spatial Wigner distribution, but we consider it in the more general Cohen's class with a proper time-frequency kernel to better process the sensor-angle relationship modeled by polynomial phase signals (PPS's) [4].

The uniformly spaced linear arrays with an inter-element spacing of half-wavelength or less achieve high resolution while avoiding spatial ambiguity. In practical applications,

This work was supported in part by the Office of Naval Research (ONR) under grant N00014-13-1-0061. however, they might be very costly due to receiver hardware and computational complexity. Sparse arrays [5]-[12] have fewer elements to achieve the same array aperture as compared to fully populated arrays. They have similar mainbeam properties and therefore provide similar performance in terms of angular accuracy, resolution, and detection of targets close to interference directions with reduced size, weight, power consumption and cost. In this paper, we also consider the effect of using sparse arrays where the array sensors are located on a grid but with missing positions. As such, the conventional time-frequency analysis tools become infeasible due to the undesirable artifacts caused from missing sensor entries [13]. Instead, we use sparse reconstruction techniques to estimate the SAD in the joint space and spatial frequency domain [13]-[15], and the obtained results are then mapped back to the estimation of source range and DOA estimation for effective source localization. The effectiveness of the proposed technique is verified using simulation results.

Notations: We use lower-case (upper-case) bold characters to denote vectors (matrices). In particular, $\mathbf{I}_{N}$ denotes the $N \times$ $N$ identity matrix. (.) ${ }^{*}$ denotes complex conjugate, whereas $(.)^{T}$ and $(.)^{H}$, respectively, denote the transpose and conjugate transpose of a matrix or vector. $\operatorname{diag}(\mathbf{x})$ represents a diagonal matrix that uses the elements of $\mathbf{x}$ as its diagonal elements. $\|\cdot\|_{2}^{2}$ implies the Euclidean $\left(l_{2}\right)$ norm of a vector. $\operatorname{Tr}($.$) denotes$ the trace of a matrix. $\mathrm{P}_{\mathrm{r}}(\cdot)$ expresses the probability density function (pdf), and $\mathcal{N}(x \mid a, b)$ denotes the random variable $x$ follows a Gaussian distribution with mean $a$ and variance $b$. In addition, $\operatorname{Re}(x)$ and $\operatorname{Im}(x)$ are the real and imaginary parts of complex element $x$, respectively. $\lfloor\cdot\rfloor$ denotes the largest integer not exceeding the argument.

\section{SyStem AND SignAl MODEL}

Consider a $K$-element linear array where the sensors are located at $p_{k} d$, with $d$ denoting the unit inter-element spacing. Let the index of the central elements to be 0 , and $p_{k} \in \mathbb{Z}$ for $k=\left\lfloor-\frac{K-1}{2}\right\rfloor, \ldots,\left\lfloor\frac{K-1}{2}\right\rfloor$. We consider a narrow-band array, i.e., the reciprocal of the bandwidth of any signals received is large compared with the propagation delay across the array. To avoid spatial ambiguity, the unit spacing $d$ is usually taken as half-wavelength, denoted as $d=\lambda / 2$.

Consider a near-field target located with a range $r$ and azimuth angle $\theta$ with respect to a reference sensor, as depicted in Fig. 1. We use the central sensor position with $p_{0}=0$ as the reference sensor. Note that a physical sensor array may or may not be present at this position. For the simplicity of the 
presentation, we only consider the single-source model in this paper, but the extension of the proposed technique to multiple target scenarios is straightforward.

In near-field environment, the range between the source and the sensors varies for each of the $K$ sensors. It is straightforward to show that the range between the source signal and sensor $k$ is given by

$$
r_{k}=\sqrt{r^{2}+p_{k}^{2} d^{2}-2 r p_{k} d \cos (\theta)} .
$$

As such, the near-field steering vector is given by

$$
\mathbf{a}=\left[1, \exp \left(j \alpha r_{1}\right), \ldots, \exp \left(j \alpha r_{K-1}\right)\right]^{T},
$$

where $\alpha=2 \pi / \lambda$, and $r_{k}$ is given in (1) for $k=$ $\left\lfloor-\frac{K-1}{2}\right\rfloor, \ldots,\left\lfloor\frac{K-1}{2}\right\rfloor$.

As such, for source signal $s(t)$, the data received at the sensor array is expressed as

$$
\mathbf{x}(t)=\mathbf{a} s(t)+\mathbf{n}(t), \quad t=1, \ldots, T,
$$

where $\mathbf{n}(t)$ is the additive Gaussian noise vector, and $T$ is the number of observed time samples.

To understand the significance of the steering vector, consider the special case of fully populated array that is uniformly spaced with interelemet spacing $d$, i.e., $p_{k}=k$. In this case, the variation over the sensors resembles a uniformly sampled frequency-modulated (FM) signal. For a moderate number of sensors, the FM signal can be considered as a PPS with a limited order. Specifically, when the range is substantially larger than the array aperture, i.e., $r \gg p_{K-1} d$, (1) can be approximated as a third-order PPS, given by

$$
r_{k} \approx r-k d \cos (\theta)+\frac{k^{2} d^{2}}{2 r} \sin ^{2}(\theta)+\frac{k^{3} d^{3}}{2 r^{2}} \cos (\theta) \sin ^{2}(\theta) .
$$

In this case, the corresponding near-field steering vector a, given in (2), is a uniformly sampled linear FM signal with respect to $k$. The corresponding spatial frequency is obtained from the derivative of phase term with respect to $k$, given as

$$
f(k)=-\frac{1}{2 \lambda}\left(r_{k+1}-r_{k-1}\right)=b_{0}+b_{1} k+b_{2} k^{2},
$$

where

$$
\begin{aligned}
& b_{0}=\frac{d}{\lambda} \cos (\theta)-\frac{d^{3}}{2 r^{2} \lambda} \cos (\theta) \sin ^{2}(\theta), \\
& b_{1}=-\frac{d^{2}}{r \lambda} \sin ^{2}(\theta), \\
& b_{2}=-\frac{3 d^{3}}{2 r^{2} \lambda} \cos (\theta) \sin ^{2}(\theta) .
\end{aligned}
$$

It is clear that the coefficients $b_{0}, b_{1}$ and $b_{2}$ are related to the range and the DOA. As such, these two parameters can then be estimated once the sensor-dependent spatial frequency is obtained. Note that, for a large range $(r \gg 1)$ such that the second-order term is negligible, $f(k)$ behaves as a chirp. Furthermore, when the source is located in a far field, as $r$ approaches infinity, $f(k)$ trends to be a constant, implying a single spatial frequency associated with a sensor-independent DOA. According to (6), the unknown range and DOA can

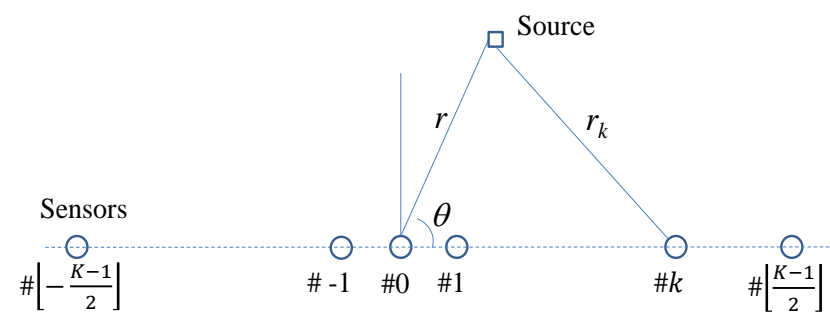

Fig. 1. The geometry for near-field source localization.

be estimated using the following expression based on the polynomial coefficients,

$$
\begin{aligned}
& \hat{\theta}=\arccos \left[\frac{\lambda}{d}\left(b_{0}-\frac{b_{2}}{3}\right)\right], \\
& \hat{r}=-\frac{d^{2}}{b_{1} \lambda} \sin ^{2}(\hat{\theta}) .
\end{aligned}
$$

Specifically, when $d=\lambda / 2$, the above expression becomes

$$
\begin{aligned}
& \hat{\theta}=\arccos \left[2\left(b_{0}-\frac{b_{2}}{3}\right)\right], \\
& \hat{r}=-\frac{d}{2 b_{1}} \sin ^{2}(\hat{\theta}) .
\end{aligned}
$$

\section{Sensor-Angle Distribution}

The SAD draws parallel to the time-frequency distribution. In essence, in SAD, we use sensor positions in lieu of time samples and the spatial frequency replaces the temporal frequency. From this analogy, SAD would help to reveal the local behavior of the spatial frequency along the array axis. The SAD corresponding to the $i$ th sensor position and spatial frequency $\xi$ at time instant $t$ is expressed as [16]

$$
\tilde{D}(i, \xi ; t)=\sum_{l=-\infty}^{\infty} \sum_{v=-\infty}^{\infty} \phi(v, l) x(i+v+l ; t) x^{*}(i+v-l ; t) e^{-j 4 \pi \xi l},
$$

where $x(i ; t)$ denotes the $i$ th element of vector $\mathbf{x}(t)$, and $\phi(v, l)$ is a kernel function that characterizes the distribution and is a function of sensor position and sensor lag. The above equation defines Cohen's class of power distribution over the joint-variable $i$ and $\xi$. In this respect, all the standard kernel designs applied in the time-frequency literature can be used with the SAD. The distribution in (9) can be averaged over the $T$ samples to reduce the effect of observation noise, yielding

$$
D(i, \xi)=\frac{1}{T} \sum_{t=1}^{T} \tilde{D}(i, \xi ; t) .
$$

Stacking $D(i, \xi)$ with respect to $i$ and $\xi$ form a sensor-angle matrix $\mathbf{D}$.

Several methods are available for the parameter estimation of linear FM and PPS (see, e.g., [17]-[19]). Such methods, however, are effective only when the samples are uniformly applied (i.e., the array is uniform linear in the underlying sensor phase progression). 


\section{SPARSe Reconstruction of SAD From Sparse ARRAY}

In this paper, we use a systematic structure consisting of uniform linear subarrays with coprime inter-element spacing. The coprime array structure was proposed in [8] for systematical sparse array configuration, and has found broad applications for DOA estimation and interference suppression [20], [21]. Benefiting from the analogy with time-frequency distributions, the random placement of array sensors is deemed to produce artifacts that clutter the SAD. Analysis of the effects of missing samples on TFD is given in [13], [14]. To avoid the induced artifacts and improve time-frequency signature estimation, sparse signal reconstruction techniques have been used and shown effective [13]-[15]. In extending these techniques to the underlying SAD problem, we first define the following sensor autocorrelation function (SAF) for time instant $t$ [16],

$$
\tilde{A}(i, l ; t)=\sum_{v=-\infty}^{\infty} \phi(v, l) x(i+v+l ; t) x^{*}(i+v-l ; t) .
$$

The averaged SAF

$$
A(i, l)=\frac{1}{T} \sum_{t=1}^{T} \tilde{A}(i, l ; t)
$$

can be similarly defined using all the $T$ time samples, along with the SAF matrix $\tilde{\mathbf{A}}$ that contains all the entries for $i$ and l. As such, the averaged SAD matrix $\mathbf{D}$ and the averaged SAF matrix A are related by a one-dimensional (1-D) Fourier transform along the lag dimension. We use the data-dependent adaptive kernel, which is based on the adaptive optimal kernel (AOK) [22] and is modified to suppress the impulsive effect due to missing samples [23]. Denote $\mathbf{a}_{i}$ as a column vector that contains all the elements in the $i$ th column of $\mathbf{A}$, and $\mathbf{d}_{i}$ as a vector corresponding to the $i$ th column of $\mathbf{D}$. Then these two vectors are related by

$$
\mathbf{a}_{i}=\mathbf{F d}_{i}, \quad i=1, \ldots, p_{k},
$$

where $\mathbf{F}$ is the discrete inverse Fourier transform matrix. Equation (13) describes a linear model relating the sparse vector $\mathbf{d}_{i}$ with compressed observation vector $\mathbf{a}_{i}$ using a Fourier dictionary. As such, $\mathbf{d}_{i}$ can be obtained by solving a sparse optimization problem, where commonly used compressive sensing techniques, like orthogonal matching pursuit (OMP) [24], can be applied. The structure-aware Bayesian compressive sensing technique can further exploit the continuity of the spatial frequency signatures for improved estimation performance [15].

As demonstrated for time-frequency analysis, the 1-D relationship between the the instantaneous autocorrelation function (IAF, the dual of SAF) and the time-frequency distribution (the dual of SAD) is much more effective than the conventional two-dimensional (2-D) approach between the ambiguity function and the time-frequency distribution [25], [26].

Once the sparse spatial frequency is estimated, the polynomial phase coefficients, i.e., $b_{0}, b_{1}$ and $b_{2}$, can be obtained using, e.g., least squares fitting. Then, the range and DOA can be estimated based on (7) or (8).

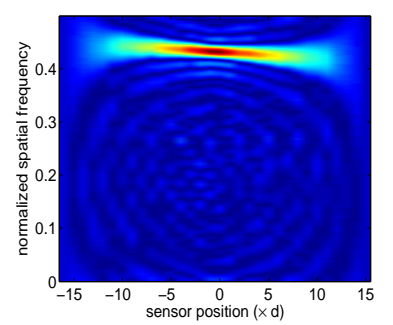

(a) WVD

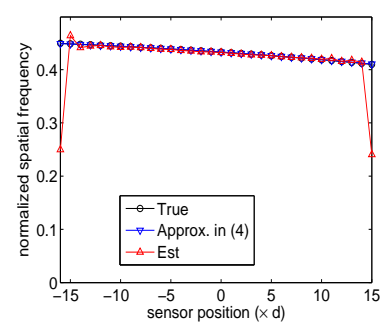

(b) Sparse reconstruction
Fig. 2. Near-field source localization using the ULA for Example 1.

\section{Simulation Results}

Simulation examples are presented to demonstrate the effectiveness of the proposed technique. Two examples are considered using a ULA as well as a sparse linear array. The first example considers the localization of a single near-field source, where the spatial frequency is approximately linear to the sensor position. The second example, we consider the situation where the source is closer to the array so that the spatial frequency is approximated as a third-order PPS with respect to the sensor positions.

Example 1. We first consider a ULA with 32 elements. The carrier frequency is considered to be $15 \mathrm{MHz}$, yielding a wavelength of $\lambda=20 \mathrm{~m}$. The interelement spacing is set to $d=\lambda / 2=10 \mathrm{~m}$. A source is located with a range of 1000 $\mathrm{m}$ and DOA of $30^{\circ}$ with respect to the reference sensor. The corresponding source coordinate is $[866.03,500] \mathrm{m}$. We use quadrature phase-shift keying (QPSK) modulated signals as an example, and 100 time samples are observed. The input signalto-noise ratio (SNR) is $0 \mathrm{~dB}$. In order to obtain a more smooth figure, we oversample the spatial frequency on a multiple of 32.

Fig. 2(a) shows the Wigner-Ville distribution (WVD) of the $\mathrm{SAD}$, which is well approximated as a chirp. Because there is no missing data, the signature can be estimated using many conventional methods as well as the proposed sparse reconstruction technique, with respect to the IAF. The corresponding sparse reconstruction results, which are depicted in Fig. 2(b), yield a high-accuracy estimation. The estimated range and DOA are $1029 \mathrm{~m}$ and $30.26^{\circ}$, and the yielding source position is $[888.8,518.5] \mathrm{m}$.

Next, we consider a sparse array configuration consisting of three uniform subarrays with coprime inter-element spacings of $M_{1}=3, M_{2}=4$ and $M_{3}=5$, as shown in Fig. 3. Compared to the 32-sensor ULA considered previously, the sparse array has 20 sensors, resulting in $37.5 \%$ of missing sensors, indicated by $\times$ in Fig. 3. As a result, the conventional PPS parameter estimation methods are not effective. In addition, the SAD is contaminated with artifacts that may obscure the real feature of the signal due to missing sensors, as illustrated in Fig. 4(a). Fig. 4(b) shows the corresponding result from sparse reconstruction, indicating a high number of erroneous spatial frequency estimations. To improve the performance, we use the AOK to suppress the effect of missing sensors, and the results are shown in Figs. 4(c) and (d). It is evident that the deteriorated effect is substantially mitigated.

We use the estimated spatial frequency signature of SAD 
$x \triangleleft x \times \triangleleft x \times \triangleleft x \times \triangleleft x \times \triangleleft \times \times \triangleleft \times \times \triangleleft \times \times \triangleleft \times \times \triangleleft \times \times \triangleleft \times \times \triangleleft$

$\begin{array}{llllllllllllllll}-16 & -14 & -12 & -10 & -8 & -6 & -4 & -2 & 0 & 2 & 4 & 6 & 8 & 10 & 12 & 14\end{array}$

(a) Subarray 1 with inter-element spacing $3 d$

$\nabla \times \times \times \Delta \times \times \times \Delta \times \times \times \Delta \times \times \times \Delta \times \times \times \Delta \times \times \times \Delta \times \times \times \Delta \times \times \times$

$\begin{array}{llllllllllllllll}-16 & -14 & -12 & -10 & -8 & -6 & -4 & -2 & 0 & 2 & 4 & 6 & 8 & 10 & 12 & 14\end{array}$

(b) Subarray 2 with inter-element spacing $4 d$

$\times \square \times \times \times \times \square \times \times \times \times \square \times \times \times \times \square \times \times \times \times \square \times \times \times \times \square \times \times \times \times \square$

$\begin{array}{llllllllllllllll}-16 & -14 & -12 & -10 & -8 & -6 & -4 & -2 & 0 & 2 & 4 & 6 & 8 & 10 & 12 & 14\end{array}$

(c) Subarray 3 with inter-element spacing $5 d$



$\begin{array}{llllllllllllllll}-16 & -14 & -12 & -10 & -8 & -6 & -4 & -2 & 0 & 2 & 4 & 6 & 8 & 10 & 12 & 14\end{array}$

(d) Array configuration

Fig. 3. The sparse array configuration.

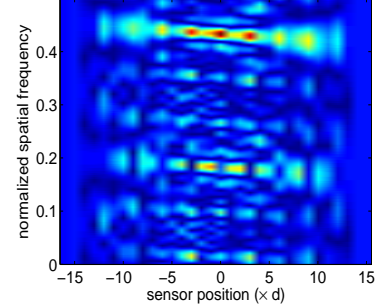

(a) WVD



(c) AOK distribution



(b) Sparse reconstruction



(d) Sparse reconstruction
Fig. 4. Near-field source localization using the sparse array for Example 1.

to obtain the coefficients $b_{0}, b_{1}$ and $b_{2}$, using least squares fitting. The range and the DOA can be estimated from Eqn. (8). We evaluate the source localization performance through Monte Carlo simulations. The average root mean square error (RMSE) of the estimated localization is used as the performance metrics, expressed as

$$
\operatorname{RMSE}=\sqrt{\frac{1}{M} \sum_{m=1}^{M}\left[\left(\Delta_{x}^{(m)}\right)^{2}+\left(\Delta_{y}^{(m)}\right)^{2}\right]},
$$

where

$$
\begin{aligned}
& \Delta_{x}^{(m)}=\hat{r}^{(m)} \cos \left(\hat{\theta}^{(m)}\right)-r \cos (\theta), \\
& \Delta_{y}^{(m)}=\hat{r}^{(m)} \sin \left(\hat{\theta}^{(m)}\right)-r \sin (\theta),
\end{aligned}
$$

and $\hat{\theta}^{(m)}$ and $\hat{r}^{(m)}$ are the estimate of $\theta$ and $r$, respectively, for the $m$ th Monte Carlo trial, $m=1, \ldots, M$. We use $M=$ 500 independent trials in all simulations. Fig. 5 compares the RMSE performance of previous example as a function of the input SNR, where 100 snapshots are used. It is evident that the source localization performance is improved as the input

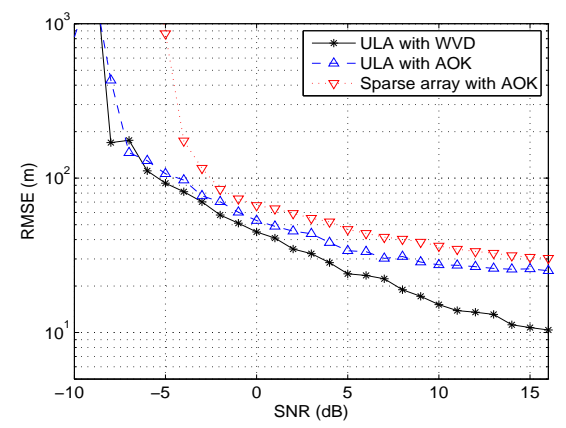

Fig. 5. RMSE versus SNR for Example $1\left(r=1000 \mathrm{~m}\right.$ and $\left.\theta=30^{\circ}\right)$.

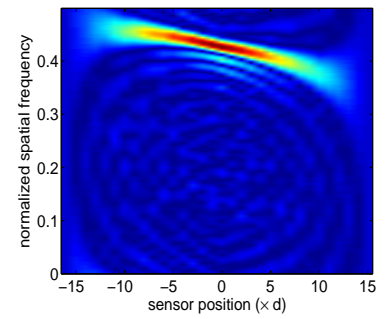

(a) WVD

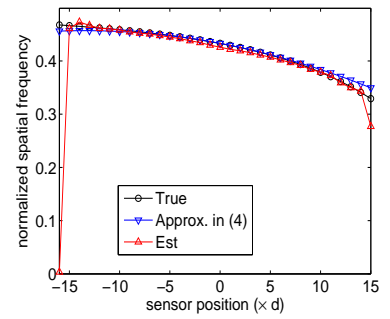

(b) Sparse reconstruction
Fig. 6. Near-field source localization using the ULA for Example 2.

increases. The WVD presents the best performance since it has been shown to be optimal in the analysis of linear FM signals [27], [28]. It is noted that, in the low SNR region, the performance of the sparse array is worse than that of the ULA with AOK processing. However, the difference becomes smaller as the SNR increases.

Example 2. In this example, the parameters are assumed to be the same as those in Example 1, but the source range is reduced to $350 \mathrm{~m}$. The WVD and the estimated spatial frequency signature using the ULA based on sparse reconstruction technique are depicted in Fig. 6, and the corresponding results using the sparse array are presented in Fig. 7. In Fig. 6 , the results show that the spatial frequency is approximated as a third-order PPS with respect to the sensor positions. Note that, for a nonlinear FM signal in the time-frequency domain, in addition to a bias of the IAF, the WVD exhibits some cross-terms. It is also noted that the model error caused by the approximation in (4) is slightly higher as the source is closer to the array, as compared to the previous example. For the sparse array, the effect of artifacts is again confirmed in 7(a) and 7(b). This effect is significantly suppressed after the AOK is applied, as shown in Figs. 7(c) and (d).

In Fig. 8, we compare the RMSE performance of Example 2. It is shown that the distribution with AOK achieves better performance because of the effective cross-term and artifact suppression. In addition, there appears a floor on the RMSE result due to its bias of IAF. For the sparse array, a similar performance is attained to that of the ULA when the input SNR is higher than $10 \mathrm{~dB}$.

\section{CONCLUSION}

In this paper, we proposed a simple near-field source localization technique using sparse arrays. We used the sensorangle distribution to characterize the sensor-dependent phase 


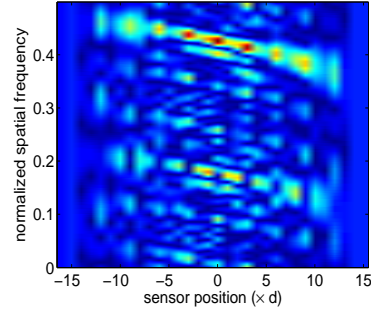

(a) WVD

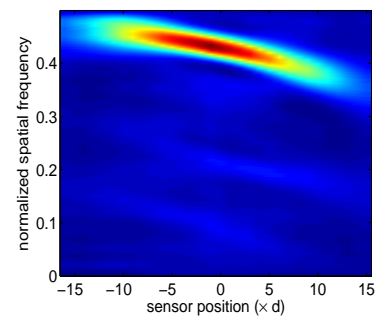

(c) AOK processing



(b) Sparse reconstruction



(d) Sparse reconstruction
Fig. 7. Near-field source localization using the sparse array for Example 2.

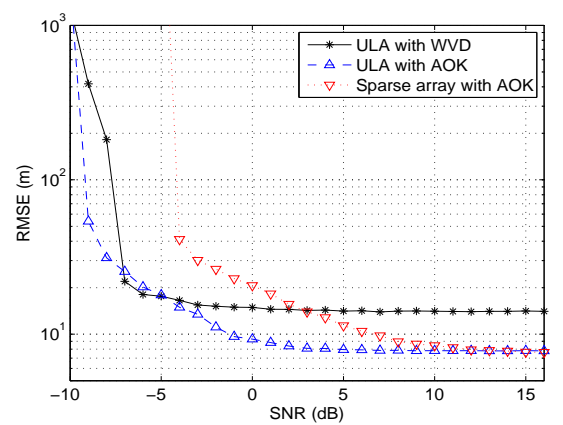

Fig. 8. RMSE versus SNR for Example $2\left(r=350 \mathrm{~m}\right.$ and $\left.\theta=30^{\circ}\right)$.

progression as a function of the source range and its direction. Sparse reconstruction techniques were used to estimate the sensor-dependent spatial frequency signature for source localization. The effectiveness of the proposed technique was verified using simulation results.

\section{REFERENCES}

[1] R. N. Challa and S. Shamsunder, "High-order subspace-based algorithms for passive localization of near-field sources," in Proc. Asilomar Conf. on Signals, Systems and Computers, Pacific Grove, CA, pp. 777-781, Nov. 1995.

[2] B. A. Obeidat, Y. Zhang, and M. G. Amin, "Range and DOA estimation of polarized near-field signals using fourth-order statistics," in Proc. IEEE ICASSP, Montreal, Canada, May 2004.

[3] B. R. Breed and T. E. Posch, "A range and azimuth estimator based on forming the spatial Wigner distribution," in Proc. IEEE ICASSP, San Diego, CA, pp. 41B.9.1-9.2, 1984.

[4] M. G. Amin, Y. Zhang, G. J. Frazer, and A. R. Lindsey, "Spatial timefrequency distributions: Theory and applications," in L. Debnath (ed.), Wavelets and Signal Processing, Birkhauser, 2003.

[5] A. Moffet, "Minimum-redundancy linear arrays," IEEE Trans. Antennas Propagat., vol. 16, no. 2, pp. 172-175, March 1968.

[6] R. T. Hoctor and S. A. Kassam, "The unifying role of the co-array in aperture synthesis for coherent and incoherent imaging," Proc. IEEE, vol. 78, no. 4, pp. 735-752, April 1990.
[7] P. Pal and P. P. Vaidyanathan, "Nested arrays: A novel approach to array processing with enhanced degrees of freedom," IEEE Trans. Signal Proc., vol. 58, no. 8, pp. 4167-4181, Aug. 2010.

[8] P. P. Vaidyanathan and P. Pal, "Sparse sensing with co-prime samplers and arrays," IEEE Trans. Signal Proc., vol. 59, no. 2, pp. 573-586, Feb. 2011.

[9] Y. D. Zhang, M. G. Amin, and B. Himed, "Sparsity-based DOA estimation using co-prime arrays, in Proc. IEEE ICASSP, Vancouver, Canada, pp. 3967-3971, May 2013.

[10] Y. D. Zhang, S. Qin, and M. G. Amin, "DOA estimation exploiting coprime arrays with sparse sensor spacing," in Proc. IEEE ICASSP, Florence, Italy, pp. 2267-2271, May 2014.

[11] S. Qin, Y. D. Zhang, and M. G. Amin, "Generalized coprime array configurations," in Proc. IEEE Sensor Array and Multichannel Signal Proc. Workshop, A Coruña, Spain, June 2014.

[12] S. Qin, Y. D. Zhang, and M. G. Amin, "Generalized coprime array configurations for direction-of-arrival estimation," IEEE Trans. Signal Proc., in press.

[13] Y. D. Zhang, M. G. Amin, and B. Himed, "Reduced interference timefrequency representations and sparse reconstruction of undersampled data," in Proc. European Signal Proc. Conf., Marrakech, Morocco, Sept. 2013.

[14] L. Stankovic, S. Stankovic, I. Orovic, and Y. D. Zhang, "Time-frequency analysis of micro-Doppler signals based on compressive sensing," in M. G. Amin (ed.), Compressive Sensing for Urban Radars, CRC Press, 2014.

[15] Q. Wu, Y. D. Zhang, and M. G. Amin, "Continuous structure based Bayesian compressive sensing for sparse reconstruction of timefrequency distributions," in Proc. Int. Conf. Digital Signal Proc., Hong Kong, China, pp. 831-836, Aug. 2014.

[16] L. Cohen, Time-Frequency Analysis, Prentice Hall, 1995.

[17] B. Friedlander and J. M. Francos, "Estimation of amplitude and phase parameters of multicomponent signals," IEEE Trans. Signal Proc., vol. 43, no. 4, pp. 917-925, 1995.

[18] B. Ristic and B. Boashash, "Comments on "The Cramer-Rao lower bounds for signals with constant amplitude and polynomial phase,', IEEE Trans. Signal Proc., vol. 46, no. 6, pp. 1708-1709, June 1998.

[19] C. Ioana, M. G. Amin, Y. Zhang, and F. Ahmad, "Characterization of Doppler effects in the context of over-the-horizon radar," in Proc. IEEE Int. Radar Conf., Washington D.C., pp. 506-510, May 2010.

[20] J. Chen, Q. Liang, B. Zhang and X. Wu, "Specturm efficiency of nested sparse sampling and co-prime sampling," EURASIP J. Wireless Commun. Netw., vol. 2013, no. 1, pp. 1-15, 2013.

[21] K. Adhikari, J. R. Buck and K. E. Wage, "Extending coprime sensor arrays to achieve the peak side lobe height of a full uniform linear array," EURASIP J. Wireless Commun. Netw., vol. 2014, no. 1, pp. 1-17, 2014.

[22] D. L. Jones and R. G. Baraniuk, "An adaptive optimal-kernel timefrequency representation," IEEE Trans. Signal Proc., vol. 43, no. 10, pp. 2361-2371, 1995.

[23] B. Jokanovic, M. G. Amin, Y. D. Zhang, and F. Ahmad, "Time-frequency kernel design for sparse joint-variable signal representations," in Proc. European Signal Proc. Conf., Lisbon, Portugal, pp. 2100-2104, Sept. 2014.

[24] J. A. Tropp and A. C. Gilbert, "Signal recovery from random measurements via orthogonal matching pursuit," IEEE Trans. Info. Theory, vol. 53, no. 12, pp. 4655-4666, 2007.

[25] P. Flandrin and P. Borgnat, "Time-frequency energy distributions meet compressed sensing," IEEE Trans. Signal Proc., vol. 58, no. 6, pp. 2974 2982, June 2010.

[26] Y. D. Zhang and M. G. Amin, "Compressive sensing in nonstationary array processing using bilinear transforms," in Proc. IEEE Sensor Array and Multichannel Signal Proc. Workshop, Hoboken, NJ, pp. 349-352, June 2012.

[27] B. Boashash and B. Ristic, "Polynomial time-frequency distributions and time-varying higher order spectra: application to the analysis of multicomponent FM signals and to the treatment of multiplicative noise," Signal Process., vol. 67, no. 1, pp. 1-23, 1998.

[28] B. Barkat and B. Boashash, "Design of higher order polynomial WignerVille distributions," IEEE Trans. Signal Proc., vol. 47, no. 9, pp. 26082611, 1999. 